\title{
Environmental Scan of the US States' and Other Countries' Notifiable Disease Provisional Data Online Display: Results and Recommendations
}

\author{
Anna Grigoryan* and Ruth Ann Jajosky \\ CDC, Atlanta, GA, USA
}

\section{Objective}

The purpose of this project was to identify ideas and potential options for an enhanced dissemination of provisional data for the US National Notifiable Diseases Surveillance System (NNDSS).

\section{Introduction}

The NNDSS is the public health surveillance system that enables all levels of public health (local, state, territorial, and federal) to monitor the occurrence and spread of the diseases and conditions that the Council of State and Territorial Epidemiologists (CSTE) has officially designated as being "nationally notifiable". The NNDSS data are a critical source of data for monitoring disease trends, effectiveness of prevention and control programs, and policy development. To provide timely NNDSS data, state and territorial health departments voluntarily report notifiable disease incidence data to CDC when they become aware of these cases and as per recommended national notification timeframes. These provisional data are published each week in Morbidity and Mortality Weekly Report (MMWR). Great strides have been made exploring and exploiting new and different sources of disease surveillance data and developing robust statistical methods for analyzing the collected data (1). However, there have been fewer efforts in the area of on-line dissemination of surveillance data, which is so important in maximizing the utility of collected data.

\section{Methods}

We conducted a search of all US State Health Department websites looking for on-line data display tables and tools for either "reportable" or "notifiable" diseases. In addition, the scope of the search of websites was expanded to include notifiable diseases of several countries, organizations and institutions. We identified examples of ways to display data that may be more effective and useful to data users than the current NNDSS data display.

Particular emphasis was placed on the display of reportable or notifiable disease provisional data. Provisional NNDSS data in MMWR Table II (2) are a running count of selected nationally notifiable infectious disease cases that have not been adjusted for variations in reporting procedures across different states or for delays in reporting. These data may include case reports considered "suspect" or "probable" for surveillance purposes in addition to those considered "confirmed". As a result, provisional data are subject to change based on the outcome of further case investigation.

\section{Results}

As of May 1, 2013 review of the US States notifiable disease websites showed that only 22 States (44\%) presented provisional data in either static report or on-line interactive data display formats. This presentation will review selected examples of provisional data display which we deemed exemplary and useful for the NNDSS.

As a result of this study we propose enhancement of current notifiable disease data display from static MMWR table format to a more interactive format. Proposed NNDSS interactive data presentation tool can help users to overcome information overload, saves users time through quick access to a database and provides opportunity to present data in a more effective visual way. Users from states will be able to compare their epidemiological findings with other states in order to plan and collaborate with each other on important public health issues. The tool may also allow several currently non-existing display options: multiple area comparisons/ mapping with user friendly options to facilitate geographical comparison across different US States, etc.

\section{Conclusions}

Advances in infectious disease informatics research in recent years has allowed significant improvements in data collection, sharing, reporting, analyzing, and data visualization, which allows better data presentation and interpretation on surveillance websites for maximizing the data usage. Our study was limited to review of available English public websites and/or databases which we could identify based on our search term strategy. In addition, this review focused on the dissemination of surveillance data, and has not reviewed the underlying surveillance systems. There may be room for improvement in the style and content of the dissemination of US nationally notifiable disease provisional data to health care professionals and the general public using interactive tools or dashboard design.

\section{Keywords}

Public Health Surveillance; Notifiable Diseases; Provisional data

\section{References}

1. Teutsch SM, Churchill RE, eds. Principles and practice of public health surveillance. 2nd edition. Oxford University Press; 2000

2. MMWR, July 19, 2013, Vol.62, No.28 http://www.cdc.gov/mmwr/pdf/ wk/mm6228md.pdf

\section{*Anna Grigoryan}

E-mail: Ffg7@cdc.gov 